\title{
Transeterification of a Mixture of Vegetable Fats with the Addition of Phytosterols
}

\author{
D. S. Honcharov, N. A. Tkachenko, and V. G. Nikolaieva
}

\section{ABSTRACT}

Pure phytosterols that are sparingly soluble in oils were subjected to a transesterification reaction using the catalyst sodium methylate in a mixture of palm stearin: palm oil: sunflower oil. As a comparison, transesterification was performed under the same conditions without the addition of phytosterols. To determine the qualitative composition of the mixture transesterified with phytosterols, analyzes were performed on the fatty acid composition, sterol composition, and melting point. The resulting product differed from the control by the presence of a characteristic odor, sterol composition, melting point.

Keywords: phytosterols, transesterification, fat-containing products, spreads, functional products, milk fat substitutes.

Submitted : February 21, 2021

Published : March 14, 2021

ISSN: $2684-1827$

DOI: 10.24018 /ejfood.2021.3.2.252

\section{S. Honcharov*}

Odessa National Academy of Food Technologies, Odesa, Ukraine.

(e-mail: dns93@ukr.net)

N. A. Tkachenko

Odesa National Academy of Food

Technologies, Odesa, Ukraine.

(e-mail: nataliya.n2013@gmail.com)

V. G. Nikolaieva

Odesa National Polytechnic University,

Odesa, Ukraine.

(e-mail: volskrad@gmail.com)

*Corresponding Author

\section{INTRODUCTION}

Phytosterols (plant sterols) are found in the cells of all plants. They are important structural components that stabilize the biological membranes of plants. In nature, sterols can be found as free sterols or as four types of conjugates in which the 3-hydroxyl group is esterified to a fatty acid or hydroxycoric acid or glycosylated by hexose (usual glucose) or 6-fatty acylhexose. Glycosides are the most common form found in cereals [7].

Phytosterols belong to the family of triterpenes: they consist of a tetracyclic structure and a side chain in position C-17 (Fig. 1). The structure of phytosterols is very similar to the structure of cholesterol, which is currently the most common steroid found in animal cells. Contrary to popular belief, plant cells also contain cholesterol, although its level usually remains below $1 \%$ of total sterols.

Unlike animal cells, several hundred different sterols have been found in plant cells. They differ primarily by substitutions in C-4 (Fig. 1) and C-24 (Fig. 1) on the side chain, the unsaturation of the side chain and rings, as well as conjugation of the alcohol hydroxyl group C-3 (Fig. 1) not only with fatty acids but also with phenolic acids and carbohydrates [9].

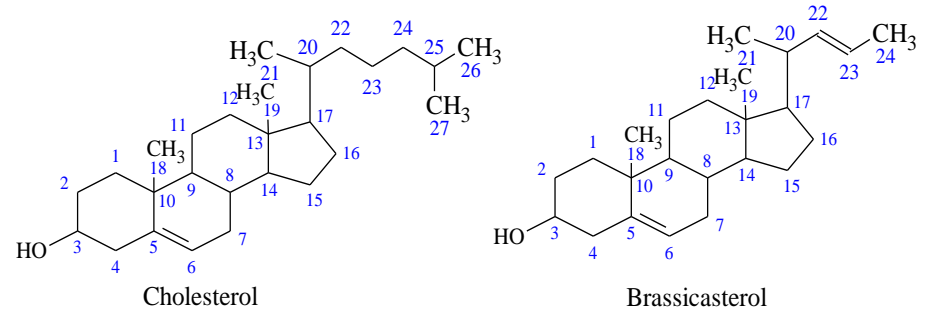

Fig. 1. The structure of sterols.

Phytosterols are a family of more than 200 different compounds derived from different species of vegetation and aquatic flora [20]. Campesterol, beta-sitosterol, and stigmasterol are the most common phytosterols, both structurally resembling cholesterol, except for the additional hydrocarbon chain at position C-24 (Fig. 1).

Phytosterols are plant sterols that have similar structural and biological functions to cholesterol [1]. They have shown protection against various chronic diseases, such as cardiovascular disease [4], hepatoprotective diabetes, and cancer [2]. The greatest results were achieved when using phytosterols in the fight against hypercholesterolemia [5]. Concerning cancer prevention, it has been stated that a diet rich in phytosterols can reduce the risk of cancer by $20 \%$ [13]. Phytosterols are structural components of the cell membrane that are involved in the regulation of membrane processes. ). The human body receives phytosterols exclusively through the food of plant origin, as the cell does not synthesize phytosterols endogenously. Serum phytosterol levels in humans range from 7 to $41 \mathrm{mmol} / \mathrm{l}(2.9-17.0 \mathrm{mg} / \mathrm{l})$. 
Plant foods, mainly nuts, seeds, vegetable oils, cereals, and legumes, are the richest source of phytosterols [1]. The group of stanols is especially distinguished, which differs somewhat in the absence of a double bond between the atoms C-5 and C-6 (Fig. 1). Plant stanols can be found in conifers such as pine and spruce. The daily dietary intake of plant stanols is about $25 \mathrm{mg} /$ day compared to sterols and in the range from 150 to $400 \mathrm{mg} /$ day, which includes $65 \%$ consumption in the form of b-sitosterol, $30 \%$ in the form of campesterol, and $5 \%$ in the form of stigmasterol [10]

There are two main periods in the history of the study and use of phytosterols. The first is characterized by an interest in phytosterols as such and their properties in the 50s of the 20th century, it was determined that phytosterols have a positive effect in normalizing cholesterol balance, they were used in pure form, but had to use fairly large doses [8]. The second stage began after the publication of data on the greater efficiency of sterol esters and glycosides in comparison with the pure substance. The esterified form of phytosterols shows good solubility in fats while the glycosidic form of phytosterols is more soluble in water. Such properties are extremely important for bioavailability, given that pure phytosterols are waxy powder with a melting point greater than $140{ }^{\circ} \mathrm{C}$ and insoluble in water and fats. One of the known methods of converting phytosterols into an esterified form is transesterification with lipase with one oil, such as rice or sunflower oil [12].

After proving the benefits of prophylactic use of phytosterols [11], foods with added phytosterols [1] have appeared on the market, including beverages, yogurts, phytosterol-containing spreads, and fat-containing products, but phytosterols can also be added to more traditional foods. clear products [6].

Esterified phytosterols can be used in the production of fatty, milk-containing products, and the presence of phytosterols changes the conditions of crystallization in water-milk fat emulsion systems. It should be noted that recent studies have shown that the presence of high amounts of phytosterols slowed down crystallization, even though at low doses phytosterols acted as centers of crystallization [3].

The purpose and objectives of the study. The work aims to study the conditions of the transesterification reaction of vegetable fats with the addition of phytosterols.

\section{MATERIALS AND METHODS}

\section{A. Materials}

For transesterification were taken food-grade palm stearin, food-grade palm oil, refined deodorized food-grade sunflower oil, 98\% phytosterols (Xi'an Sgonek Biological Technology Co., Ltd. China), sodium methylate, citric acid.

\section{B. Experimental Installation for Transesterification}

For transesterification, a 3-necked flask was used in a sand bath with a vacuum, a thermometer, and a magnetic stirrer.

\section{Experiment 1}

A portion of palm stearin: palm oil: sunflower oil in a ratio of 75:10:15 heated to melting point and poured into a reaction flask then stirred for 10 minutes, a portion of $0.25 \mathrm{~g}$ of sodium methylate was added, the reaction was carried out for 30 minutes, then $0.01 \mathrm{~g}$ of citric acid was added to neutralize sodium methylate. After completion of the reaction, the hot mixture was purified from the catalyst and citric acid by filtration under vacuum through a paper filter with a pore size of $8 \ldots 12 \mu \mathrm{m}$. The resulting product below will be Sample 1 .

\section{Experiment 2}

A portion of palm stearin: palm oil: sunflower oil in a ratio of 75:10:15 heated to melting point and poured into a reaction flask then stirred for $10 \mathrm{~min}$, a portion of $0.25 \mathrm{~g}$ of sodium methylate was added, $6 \%$ of phytosterols were added after stirring with sodium methylate. The reaction was carried out for $30 \mathrm{~min}$, then $0.01 \mathrm{~g}$ of citric acid was added to neutralize sodium methylate. After completion of the reaction, the hot mixture was purified from the catalyst and citric acid by filtration under vacuum through a paper filter with a pore size of $8 \ldots 12 \mu \mathrm{m}$. The resulting product hereinafter referred to as Sample 2 .

\section{E. Determination of $\mathrm{Na}$}

The analyzed sample was subjected to mineralization with a solution of nitric acid in the system of microwave decomposition "Mileston" ethos easy. After decomposition, the mineralization was quantitatively transferred to a volumetric flask and brought to the mark with water. An atomic absorption spectrometer with electrothermal atomization of high resolution "Analytik Jena" ContrAA $800 \mathrm{G}$ was used for analysis. calibration of the sample was performed by the method of standard additives.

\section{F. Determination of Fatty Acid Composition}

The sample was transferred into a test tube, isooctane was added, the contents were shaken. Then a solution of potassium methylate was added and the contents were shaken again. After settling the sample, the upper layer was selected for further analysis. For analysis, a gas chromatograph with a flame ionization detector "Shimadzu" GC-2010 Plus was used. Capillary column - "Thermo" TR-FAME 30m. Helium carrier gas. Evaporator - with flow separation.

\section{G. Determination of Sterol Composition}

The sample, with added betulin solution, was subjected to hydrolysis in $\mathrm{KOH}$ solution. After hydrolysis, ethyl alcohol was added to the sample. The mixture was then passed through a column filled with alumina and eluted with ethyl alcohol and diethyl ether. The collected fraction, after column chromatography, was evaporated on a rotary evaporator to a drop, then in a stream of nitrogen to dryness. The dry residue from the walls of the flask was washed with diethyl ether and applied to TLC plates with a layer of silica gel. The dried plates were placed in a chamber with a mixture of hexane: diethyl ether 1: 1 . After raising the front to the top of the plate, they were pulled out and dried. The plates were sprinkled with methanol and marked White line, $1.5-2 \mathrm{~cm}$ wide, which corresponds to sterols and betulin. The sorbent layer was cleaned from the plate along the specified line and washed with diethyl ether. Then the ether was evaporated to dryness in a stream of nitrogen. The dry residue was washed with small portions of diethyl ether in a vial in which it was evaporated to dryness. A silylating agent (N-Methyl-Ntrimethylsilylhepta-fluoro butyramide) was added to the dry residue. The vial was thermostated at 105 degrees Celsius. 
After cooling, isooctane was added to the contents in an amount equivalent to the silylating agent. The contents were mixed and subjected to further analysis. A gas chromatograph with a Shimadzu flame ionization detector GC-2010 Plus was used for analysis. Capillary column - "Agilent" SE-54. Helium carrier gas. Evaporator - with flow separation.

\section{H. Determination of the Melting Point}

To determine the melting point in the samples using the method for determining the flow point with polymorphism. In the capillaries with a diameter of $1.3 \mathrm{~mm}$ was taken a sample, which was defended for 17 hours at a temperature of 20 degrees so that it was filled with $10 \mathrm{~mm}$, the excess particles of the sample were removed from the surface of the capillary. After that, the capillaries were attached to the thermometer so that the sample was at the level of the thermometer ball. The capillary thermometer was immersed in cold water, which was stirred and heated to 1 degree/min. The melting point was determined by the temperature at which fat starts going up.

\section{RESULTS AND DISCUSSION}

The main indicators of milk fat substitute are neutral taste and smell - to obtain a safe substitute of the highest quality, the following raw materials are selected: palm kernel oil (similar in characteristics to coconut oil), palm stearin (used as a substitute for solid milk fats in oil and fat production) and sunflower oil (to improve the taste so that the composition does not leave a salty taste). Palm oil was not included in the composition of the fat composition due to its consistency properties at $20^{\circ} \mathrm{C}$, according to DSTU 4306: 2004 it is semisolid and mobile, which does not allow to fully regulate the melting temperature.

Studies of the obtained product showed that sample 1 and sample 2 have a homogeneous structure, white color, sample 2 has a shade of characteristic aroma and taste of phytosterols. That is, we see that the addition of phytosterols did not have a significant effect on the appearance of the product.

Determination of residual sodium was performed to determine the amount of catalyst remaining in the samples. In sample 1 , the result was $3,112 \mathrm{mg} / \mathrm{kg}$ or $0.0257 \%$ or in terms of sodium methylate $0.06 \%$. In sample 2 , the result was $2.242 \mathrm{mg} / \mathrm{kg}$, or $0.0183 \% 0.0257 \%$ or in terms of sodium methylate $0.042 \%$. Given that the reaction was taken $0.25 \%$, we can say that in sample 1 there was $10.3 \%$ of the initial amount, and in sample $27.3 \%$ of the initial amount.

In terms of fatty acid composition, samples 1 and 2 are similar, which means that the addition of sterols to the transesterification reaction did not affect the fatty acid composition and did not cause the transition of cis acids into trans forms. (Table 1).

Examination of the sterol composition of the obtained fat mixture enriched with phytosterols by chromatographic method indicates the presence of $2.89 \pm 0.01 \%$ of phytosterols, while the transesterified mixture without the addition of extraneous sterols was in the composition of $0.11 \pm 0.01 \%$ of phytosterols. The obtained results allow us to conclude that during transesterification $48 \%$ of phytosterols were converted into fat-soluble form. This effect can be caused by the simultaneous esterification of phytosterols and changes in fatty acids during transesterification. Given that the phytosterols remaining on the filter did not react are quite specific and have a relatively high cost, they should be collected and sent for a second reaction cycle by adding to pure phytosterols.

\begin{tabular}{ccc}
\multicolumn{3}{c}{ TABLE 1. FATTY ACID COMPOSITION OF TRANSESTERIFIED SAMPLE } \\
\hline Name of fatty acid & Sample 1 & Sample 2 \\
\hline C 10:0 Capric, $\%$ & 0.26 & 0.22 \\
C12:0 Lauric, \% & 4.08 & 3.72 \\
C13:0 Tridecanoic, \% & 0.01 & 0.01 \\
C 14:0 Myristic, \% & 2.24 & 2.12 \\
C 15:0 Pentadecanoic, $\%$ & 0.04 & 0.04 \\
C16:0 Palmitic, \% & 42.65 & 42.60 \\
C16:1 Palmitoleic, \% & 0.09 & 0.09 \\
C17:0 Heptadecanoic, \% & 0.09 & 0.09 \\
C17:1 Cis-10 Heptadecenoic, $\%$ & 0.02 & 0.02 \\
C18:0 Stearic, \% & 4.50 & 4.57 \\
Tr 18:1 Elaidic, \% & 0.33 & 0.34 \\
C 18:1 n9c Oleic, \% & 30.09 & 29.99 \\
Tr 18:2 Octadecadienoic, \% & 0.04 & 0.05 \\
C18:2 Linoleic, \% & 14.71 & 15.26 \\
C18:3n6 Gamma-linolenic, \% & 0.32 & 0.32 \\
C18:3 Alfa-linolenic, \% & 0.09 & 0.09 \\
C 20:1 n9 Icosenoic, \% & 0.12 & 0.12 \\
C 24:0 Lignoceric, \% & 0.09 & 0.10 \\
\hline
\end{tabular}

Analyzing the chromatograms of the sterol composition, we can see the difference between the sterol composition (Table 2) of sample 1 and sample 2 due to the addition of the sterol fraction, which still contains a large amount of sitosterol and campesterol.
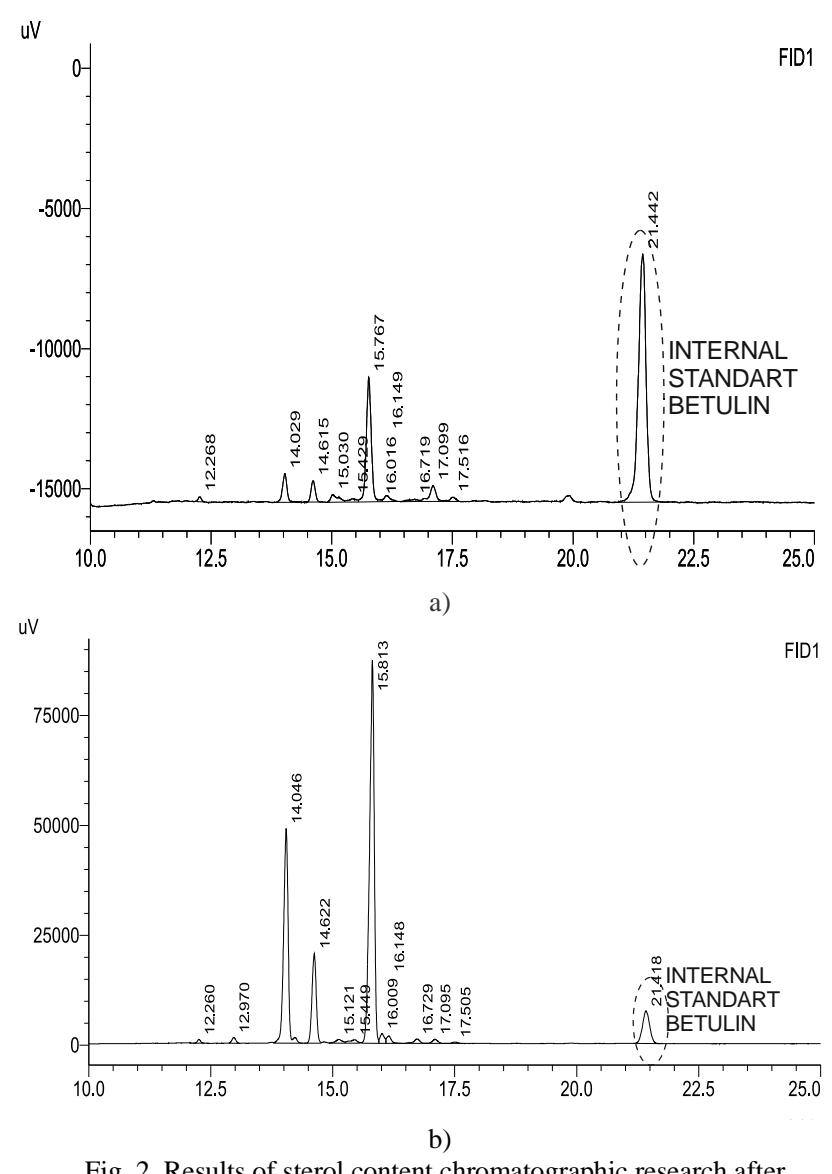

Fig. 2. Results of sterol content chromatographic research after transesterification without phytosterols (a) and by adding pure phytosterols (b).

The results of measuring the melting point showed that when phytosterols are added, the melting point decreases from 36 to $32{ }^{\circ} \mathrm{C}$, which is caused by the presence of 
phytosterol esters of fatty acids, which increase the plasticity of the fat mixture. This aspect is the most important, because the melting point is a qualitative indicator of milk fat substitutes, affects the consistency, taste, and storage conditions.

TABLE 2: STEROL CONTENT OF TRANSESTERIFIED PRODUCTS

\begin{tabular}{ccc}
\hline Name of sterol component & Sample 1 & Sample 2 \\
\hline Cholesterol, \% & 1.6 & 0.3 \\
Brassicasterol, \% & 0.0 & 0.0 \\
Campasterol, \% & 12.0 & 29.2 \\
Stigmasterol, \% & 8.1 & 11.3 \\
Delta-7-Campsterol, \% & 5.3 & 0.8 \\
Clerosterol, \% & 1.9 & 0.9 \\
Sitosterol, \% & 53.7 & 53.0 \\
Sitostanol, \% & 0.5 & 1.4 \\
Delta-5-Avenasterol, \% & 3.4 & 1.1 \\
Delta 5,24 stigmadienol, \% & 2.0 & 0.9 \\
Delta-7-stigmastenol, \% & 9.3 & 0.8 \\
Delta-7-avenasterol, \% & 2.2 & 0.3 \\
Total content in sample, ppm & 1150 & 28900 \\
\hline
\end{tabular}

Approbation of research results. The research results allow modernizing the existing transesterification technologies for the production of transesterified fat with new properties. The fat transesterified by this technology can be used for a wide range of fat-containing products, in particular spreads, sauces, sour cream products.

\section{CONCLUSION}

During transesterification, a mixture of fats with phytosterols was obtained, which can then be used for the manufacture of fat-containing foods. The product thus obtained can be on a par with traditional milk fat substitutes, although due to the transesterification technology there are no or have to trace trans isomers of fatty acids, and the presence of phytosterols in an accessible form gives special dietary qualities to the product.

Conversion of green fat with added phytosterols as a promising technology for fatty products, supplemented with phytosterols. The price is tied to this mode of esterification to be kept immutable, and from the technological point of view, it can be known that the filter of the fine product does not need to be processed, but at the same time there is an excess of phytosterol in the filter that can be recycled.

\section{REFERENCES}

[1] M. J. Lagarda, G. García-Llatas, i R. Farré, «Analysis of phytosterols in foods», J. Pharm. Biomed. Anal., vol. 41, vol. 5, p. 1486-1496, Cep 2006, doi: 10.1016/j.jpba.2006.02.052.

[2] R. A. Moreau et al., «Phytosterols and their derivatives: Structural diversity, distribution, metabolism, analysis, and health-promoting uses», Prog. Lipid Res., vol. 70, p. 35-61, 2018, doi: 10.1016/j.plipres.2018.04.001.

[3] D. S. Honcharov and N. A. Tkachenko, "PROSPECTS OF THE USE OF PHYTOSTEROLS IN THE PRODUCTION OF PRODUCTS FOR HEALTHY NUTRITION", Scientists Notes of TNU named after VI Vernadsky Technical Series. 30, issue 69, p. 150-157, 2019. [

4] L. Calpe-Berdiel, J. C. Escolà-Gil, i F. Blanco-Vaca, «New insights into the molecular actions of plant sterols and stanols in cholesterol metabolism», Atherosclerosis, vol. 203, vol. 1, p. 18-31, 2009, doi: 10.1016/j.atherosclerosis.2008.06.026.

[5] F. Blanco-Vaca, L. Cedó, i J. Julve, «Phytosterols in Cancer: From Molecular Mechanisms to Preventive and Therapeutic Potentials», Curr. Med. Chem., vol. 26, vol. 37, p. 6735-6749, 2019, doi: $10.2174 / 0929867325666180607093111$.
[6] J. J. A. Ferguson, E. Stojanovski, L. MacDonald-Wicks, i M. L. Garg, «Curcumin potentiates cholesterol-lowering effects of phytosterols in hypercholesterolaemic individuals. A randomized controlled trial», Metabolism, vol. 82, p. 22-35, 2018, doi: 10.1016/j.metabol.2017.12.009.

[7] V. R. Ramprasath i A. B. Awad, «Role of Phytosterols in Cancer Prevention and Treatment», J. AOAC Int., vol. 98, vol. 3, p. 735-738, 2015, doi: 10.5740/jaoacint.SGERamprasath.

[8] N. Shahzad et al., «Phytosterols as a natural anticancer agent: Current status and future perspective», Biomed. Pharmacother., vol. 88, p. 786794, 2017, doi: 10.1016/j.biopha.2017.01.068.

[9] R. A. Moreau, B. D. Whitaker, i K. B. Hicks, «Phytosterols, phytostanols, and their conjugates in foods: structural diversity, quantitative analysis, and health-promoting uses», Prog. Lipid Res., vol. 41, vol. 6, p. 457-500, 2002, doi: 10.1016/S0163-7827(02)000061 .

[10] S. Negishi, I. Hidaka, I. Takahashi, i S. Kunita, «Transesterification of phytosterol and edible oil by lipase powder at high temperature», J. Am. Oil Chem. Soc., vol. 80, vol. 9, p. 905-907, 2003, doi: 10.1007/s11746003-0794-x.

[11] J. QuÍlez, P. Garcĺa-Lorda, i J. Salas-Salvadó, «Potential uses and benefits of phytosterols in diet: present situation and future directions», Clin. Nutr., vol. 22, vol. 4, p. 343-351, 2003, doi: 10.1016/S02615614(03)00060-8.

[12] D. Kouzounis, A. Lazaridou, i E. Katsanidis, «Partial replacement of animal fat by oleogels structured with monoglycerides and phytosterols in frankfurter sausages», Meat Sci., vol. 130, p. 38-46, 2017, doi: 10.1016/j.meatsci.2017.04.004.

[13] L. M. Zychowski et al., «Effect of Phytosterols on the Crystallization Behavior of Oil-in-Water Milk Fat Emulsions», J. Agric. Food Chem., vol. 64, vol. 34, p. 6546-6554, 2016, doi: 10.1021/acs.jafc.6b01722. 